\title{
CORRELATION BETWEEN BULL FERTILITY AND SPERM CELL VELOCITY PARAMETERS GENERATED BY COMPUTER-ASSISTED SEMEN ANALYSIS
}

\author{
Ádám NAGY ${ }^{1 *}$, Tassos PolichronOPOUlos ${ }^{1}$, András GÁSPÁRDY $^{2}$, László SOLTI ${ }^{1}$ \\ and Sándor $\mathrm{CSEH}^{1}$ \\ ${ }^{1}$ Department and Clinic of Reproduction, ${ }^{2}$ Department for Animal Breeding and \\ Nutrition, Faculty of Veterinary Science, Szent István University, \\ István u. 2, H-1078 Budapest, Hungary
}

(Received 31 October 2014; accepted 22 June 2015)

\begin{abstract}
Motility is one of the most important characteristics associated with the fertilising ability of spermatozoa indicating their viability and structural integrity. Therefore, the examination of motility constitutes an integral part of semen analysis. Computer-assisted semen analysis (CASA) allows an accurate and objective assessment of different sperm motion characteristics with high repeatability. The aim of this study was to evaluate the different kinematic (velocity) parameters of frozen/thawed bull semen and determine if any of them could be correlated with their fertilising capability after insemination based on the achieved pregnancy rate. Ejaculates from 10 bulls were collected and frozen. The kinematic/velocity parameters of spermatozoa were measured by CASA and compared to the pregnancy results of almost 9,000 females artificially inseminated (AI) with frozen semen of any of the 10 tested bulls. The data of the experiments are summarised mainly with a focus on the effects of individual velocities (curvilinear velocity: VCL, straight-line velocity: VSL, average path velocity: VAP) on fertility rather than on the influence of progressive motility as a whole. We conclude that VAP is the most useful semen motility characteristic which has clinical relevance in the prediction of fertility.
\end{abstract}

Key words: Semen evaluation, CASA, fertility prediction, velocity characteristics, semen motility

Male fertility is an important factor in bovine reproduction, considering that the semen of any particular bull will be used for the insemination of several females. Fertility evaluation of males is primarily based on quality assessment of semen using conventional parameters such as morphology, concentration and motility of spermatozoa. The correlation between semen quality and fertility has been well documented (Bratton et al., 1956; Davis et al., 1987; Gerris and Khan, 1987; Amann, 1989; Marshburn et al., 1992; Amann and Hammerstedt, 1993;

*Corresponding author; E-mail: Nagy.Adam@aotk.szie.hu; Phone: 0036 (1) 478-4203; Fax: 0036 (1) 478-4207 
Gunzel-Apel et al., 1993; Irvine et al., 1994; Koops et al., 1995; Christensen et al., 1999; Kathiravan et al., 2008; Freour et al., 2012). Sperm motility is undoubtedly essential for fertilisation both in vivo and in vitro. Motility is indispensable for successful sperm transport, a step that can partly be bypassed by in vitro fertilisation. Thus, examination and determination of sperm motility is a significant part of semen quality evaluation since it is commonly regarded as one of the most important characteristics associated with the fertilising ability of spermatozoa.

Until recently, the assessment of sperm motility was based solely on subjective microscopic evaluation of the total proportion of motile spermatozoa with results varying between $30 \%$ and $80 \%$ (Barros et al., 1973; Budworth et al., 1988; Amann, 1989; Chan et al., 1989; Auger et al., 1993; Broekhuijse et al., 2012). Due to the large variations observed, comparability of the results obtained by different laboratories is questionable (Dott and Foster, 1979). Recently, increasing attention has been paid to the objective evaluation and characterisation of sperm movement rather than simply determining the total proportion of motile spermatozoa. The aim of computer-assisted semen analysis (CASA) is to provide objective values for sperm motility more rapidly and accurately than by traditional methods (visual observation). Computer-assisted semen analyzers provide precise information on different sperm motion/velocity characteristics (Amann and Waberski, 2014). The use of CASA allowed the identification of different age-related decreases in velocity parameters of young men with proven fertility (Freour et al., 2012).

CASA was first proposed 35 years ago by Dott and Foster and nowadays is used routinely in most human andrology centres (Dott and Foster, 1979). However, the increasing number of recent publications concerning CASA in veterinary medicine and research demonstrates the need for objective methods to assess animal semen quality, including motility (Verstegen et al., 2002). AI centres started to adopt CASA technology in order to increase the accuracy of their quality evaluation process connected with motility analyses.

Several CASA systems are marketed for veterinary use, but these differ greatly according to individual manufacturers based on optic, software and algorithms used for identification, classification and trajectory reconstruction of different sperm motion characteristics, making it very difficult to compare the results between laboratories (Marshburn et al., 1992; Krause, 1995; Coetzee et al., 1999). Both the vendor and the user of any CASA system would have to provide adequate data about reliability and validation, therefore the World Health Organization suggests specific guidelines for use of CASA instruments in order to obtain reliable and repeatable results (World Health Organization, 2010). Unfortunately, similar guidelines do not exist in veterinary clinical and research practice, therefore every institution uses either the WHO guidelines or their own settings based on previous experience. 
The main objective of our experiment was to summarise the results of the effects of individual velocities (curvilinear velocity: VCL, straight-line velocity: VSL, average path velocity: VAP) on fertility as this is defined by the NRR30 (non-return rate on day 30) and PR75 (pregnancy rate on day 75) to reflect the motion parameters of each spermatozoon travelling through a microscopic field, rather than just progressive motility as a whole. We compared the strength of the relationships between different velocity parameters and sperm fertility.

\section{Materials and methods}

\section{Bull semen samples}

Frozen semen samples of Holstein-Friesian breeding bulls (3-4 years old; $\mathrm{n}=10$ ) were produced in $0.25-\mu 1$ straws in an artificial insemination centre (Gödöllö, Hungary). Each straw contained approximately 25 million spermatozoa. The semen samples were frozen with the same routine cryopreservation technology using the same extender. Cows (about 9000) were inseminated with semen originating from the same batch of tested samples in 143 different dairy farms. Artificial inseminations (AI) were carried out by well-trained local technicians of the farms.

The fertility data in the form of non-return rate in 30 days (NRR30) as defined by regular heat observations (three times a day) and pregnancy rate on day 75 (PR75) as defined by rectal palpation, were obtained after the rest of the batch was dispensed and used for $\mathrm{AI}$ in different farms. The AIs were performed in the spring of 2010 .

\section{Semen evaluation}

For semen evaluation the MedeaLab CASA Version 4.1 (Germany) was used that is capable of evaluating not only the motility but also the morphology of spermatozoa. It uses sperm morphology characteristics and flagellum detection in order to recognise accurately sperm cells and exclude debris (epithelial cells, extender particles, etc.) that might be present in the fresh or frozen ejaculate, making the actual evaluation more accurate and overcoming problems reported elsewhere (Andersson et al., 1992).

The undiluted semen samples were examined immediately after thawing. MedeaLab CASA Version 4.1 is capable of evaluating accurately the motility of the spermatozoa up to a concentration of $120 \times 10^{6} / \mathrm{mL}$. An Olympus BX-41 phase contrast microscope with a high-resolution camera (SPT-M308CE, Sony, Japan) was used for the evaluation of semen samples with a final magnification of $\times 200$. We also used a heated stage $\left(37^{\circ} \mathrm{C}\right)$ and every measurement was carried out by the same person. A video recorder was also connected to the micro- 
scope to record the image for future reference. For every sample, a total of 10 fields were examined from two different disposable $20 \mu \mathrm{m}$ depth capillary chambers (CellVision, Germany; loading volume: $7 \mu$, concentration: $40-50 \times 10^{6} / \mathrm{mL}$ ) giving an evaluation of approx. 1500 cells per sample. Each field was recorded for $8 \mathrm{sec}$ (total of $80 \mathrm{sec}$ per sample) and the results consisted of velocity parameters VAP, VSL and VCL.

All data were stored in the system database and detailed records were kept together with the videotaped samples for the correlation of semen characteristics of the samples with the pregnancy results in order to draw conclusions about the fertility.

During the evaluation, the instrument settings were as follow: tracking thresholds set to 185 , morphology thresholds set to 130 , automatic tracking objects set to 50 , search position 1000 radius (pixel) 20, stop width 2 , match factor 2 , exclude min length (frames) 10 . Standard video image acquisition rates were used which is typically $50 \mathrm{~Hz}$ in the PAL system.

\section{Statistical analysis}

Chi-square analysis was applied in order to prove significant differences in the frequency (or nonparametric) characteristics between the groups (NRR30 and PR75) by the evaluations. Single trait one-way analysis of variance (ANOVA) was applied for the parametric characteristics (VCL, VSL, VAP) and the least significant difference (LSD), F-values and probabilities were calculated (STATISTICA 6.0, StatSoft, Inc. 2003). Linear correlation coefficient was applied in order to evaluate the relationship between the fertility data as they are expressed by the non-return rates (NRR30, PR75) for 30 days and 75 days. Regression analysis was also used to determine the connection between VAP and non-return rates in order to predict bull fertility based on VAP values.

\section{Results}

During the experiment, pregnancy data were obtained from 8909 inseminations on more than 100 farms with a total of 6590 animals being positive for pregnancy on day 30 and 4525 animals found positive for pregnancy on day 75 (Table 1).

Using the above data and a linear correlation coefficient we evaluated the relationship between fertility rate on NRR30 and PR75 with a resulting value of $r$ $=0.975(\mathrm{P}<0.001)$. Chi-square analysis for these data revealed a value of $47.763(\mathrm{P}<0.01)$ for NRR30 and a value of $41.191(\mathrm{P}<0.01)$ for PR75.

During semen evaluation an average of 2330 spermatozoa were tracked per bull. This experiment focused mainly on the actual individual velocities rather than progressive motility per se. The parameters investigated were VCL, 
Table 3

Individual velocity correlations with non-return rates

\begin{tabular}{ccc}
\hline & NRR30 & PR75 \\
\hline VCL & 0.6671 & 0.6300 \\
& $\mathrm{P}=0.035$ & $\mathrm{P}=0.051$ \\
VSL & 0.6669 & 0.6390 \\
& $\mathrm{P}=0.035$ & $\mathrm{P}=0.047$ \\
VAP & 0.7029 & 0.6652 \\
& $\mathrm{P}=0.023$ & $\mathrm{P}=0.036$
\end{tabular}

Sample size: NRR30: 6590; PR75: 4525. NRR30: non-return rate at day 30, PR75: pregnancy rate at day 75

From the statistical analysis a close correlation with high significance was found for each of the velocities (VCL, VSL, VAP) which is evident for both pregnancy rates (NRR30 and PR75) (Table 3). Using the above velocities and NRR30 as well as PR75 data, we plotted the data on two different graphs (Figs 1 and 2).

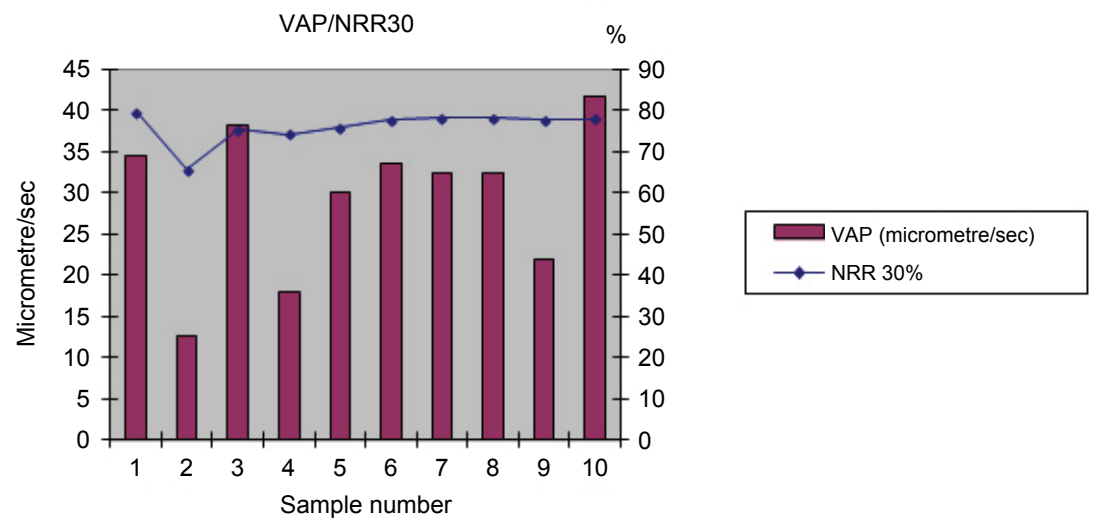

Fig. 1. Graph of average path velocity over non-return rate at day 30 for each sample. NRR30: non-return rate at day 30, VAP: average path velocity

We found that by using the equation NRR30 $=47.9615+19.5364 \times \log _{10}$ (X) where $\mathrm{X}$ is the average VAP of the sample in question, the fertilising potential of a frozen bull semen can be predicted as this is expressed by the NRR30 with acceptable accuracy when the dataset is similar to the dataset used for this study. Alternatively, using the equation PR75 $=11.0784+30.2738 \times \log _{10}(\mathrm{X})$ we can more accurately predict the pregnancy rate (Fig. 3 ). 


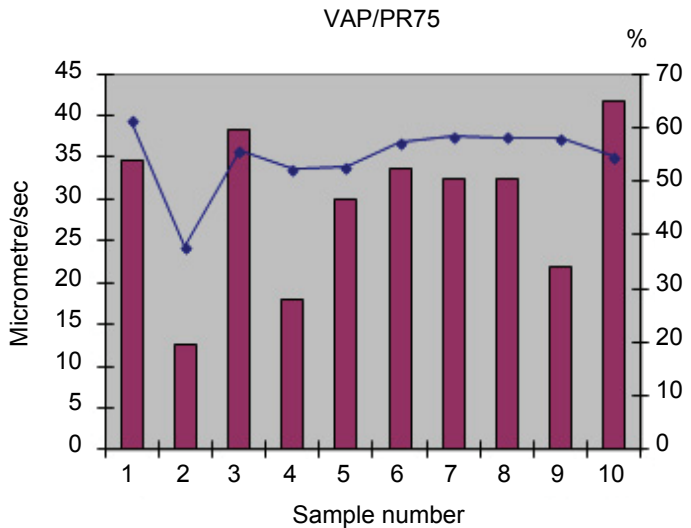

$\square$ VAP (micrometre/sec) $\multimap$ PR $75 \%$

Fig. 2. Graph of average path velocity (VAP) over pregnancy rate at day 75 for each sample. PR75: pregnancy rate at day 75 , VAP: average path velocity

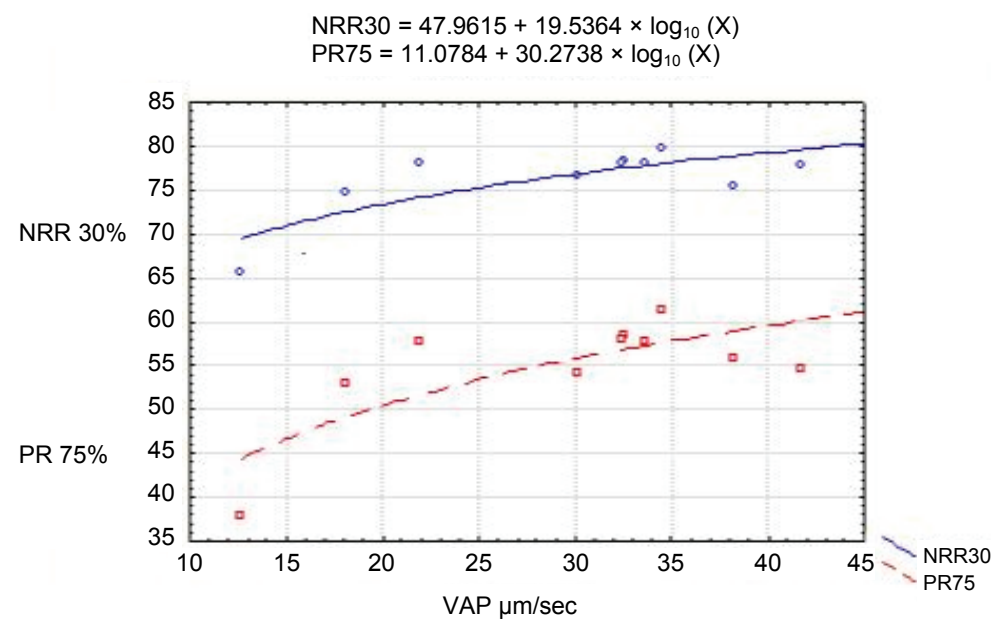

Fig. 3. Logarithmic plot of average path velocity over non-return rate at day 30 and pregnancy rate at day 75. NRR30: non-return rate at day 30, PR75: pregnancy rate at day 75, VAP: average path velocity (designated ' $\mathrm{X}$ ' in the equations)

\section{Discussion}

Significant differences were observed in motility parameters between sperm that resulted in a high fertilisation rate and those that failed to fertilise oocytes in vitro (Bollendorf et al., 1996; Donnelly et al., 1998; Hirano et al., 2001). When the traditional subjective visual evaluation system was used to assess sperm motility and the results were compared to fertility, a contradictory relationship was found between them (Andersson et al., 1992; Marshburn et al., 1992; Zhang et al., 1998; Januskauskas et al., 1999). Several studies demon- 
strated that the traditional microscopic visual technique for the determination of motile sperm ratio is inaccurate, imprecise and its repeatability is very low (Gunzel-Apel et al., 1993; Centola, 1996; Coetzee et al., 1999; Larsen et al., 2000; Broekhuijse et al., 2011). There is a lack of evidence-based information about the relationship between motility/velocity characteristics and the fertilising ability of bull spermatozoa; therefore, it is unknown whether sperm fertility could really be predicted by these parameters. The lack of a significant relationship between motility and fertility might be attributed to several factors including variation between individual bulls or the presence of excess/adequate number of spermatozoa in the insemination dose. Since CASA provides special sperm velocity/motility parameters, it gives more valuable information on the physiological status of spermatozoa.

CASA offers potential for a more accurate prediction of fertility compared to the traditional microscopic semen evaluation technique (den Daas, 1992; Christensen et al., 1999; Broekhuijse et al., 2012). Several attempts have been made to correlate different motion characteristics of bull spermatozoa with oocyte penetration rate, in vitro fertility rate and field fertility (den Daas, 1992; Kathiravan et al., 2008). Budworth et al. (1988) observed a significant correlation between ratio and velocity of motile sperm and fertility. In an early experiment using frozen-thawed bull semen, Amann (1989) observed a correlation between motility/velocity characteristics (total motility rate, VCL, VSL, LIN and $\mathrm{BCF}$ ) and fertility. In contrast to this finding, in their recent publication Amann and Waberski (2014) suggest that the evaluation of sperm motion cannot accurately predict the fertilising potential of sperm in a given sample when used for AI. They, however, recommend reliance on curvilinear velocity (VCL) and straight-line velocity STR (VSL/VAP) for each spermatozoon with categorisation into three groups: (1) immotile, VCL near zero; (2) undesirable, low VCL or STR; and (3) satisfactory, VCL and STR meet standards. Farrell et al. (1998) reported very high correlation between motility parameters evaluated by CASA on fresh semen and bull fertility with quite good prognostic value (fertility was defined as 59 days of non-return rate). Oliveira et al. (2013) recently found the same high correlation between multiple combinations of CASA parameters and bull fertility.

The situation is quite similar in the field of human reproduction, considering that Fetterolf and Rogers (1990) observed that motility and velocity data generated by CASA correlated and predicted very well the outcome of human sperm penetration assay. Larsen et al. (2000) found that motility data (percentage of motile spermatozoa, concentration of motile spermatozoa) obtained by CASA measurements can be used for the prediction of fertility potential in normal men. Experimental results obtained in pigs indicate that the CASA motility pattern makes it possible to discriminate between the fertilising capacity of ejaculates (Broekhuijse et al., 2012). Hirano et al. (2001) found a very close relationship 
between sperm motility characteristics assessed by CASA and in vitro fertilisation rates in humana. The same was found in animal model experiments (Verstegen et al., 2002).

In this study not the fresh but rather the frozen-thawed bull semen motility parameters were evaluated since that was the actual semen used for AI. From the investigation of weighted values of the non-return rates (Table 1) we found significant differences among bulls [NRR30 $\mathrm{Chi}^{2}=47.763(\mathrm{P}<0.001)$ and PR75 $\left.\mathrm{Chi}^{2}=41.191(\mathrm{P}<0.001)\right]$. The reasons responsible for these differences are assumed to be multifactorial and separate from the semen fertilising ability per se, which was the investigated parameter in this experiment. The animals were proven to be pregnant by day 75 post-AI by rectal palpation and only suggestive of pregnancy by day 30 post-AI as it was evident by heat observation.

Our results are in harmony with those of Farrell et al. (1998) who found that CASA provides repeatable estimates of many criteria of sperm movement/ velocity parameters which correlates well with the fertility potential of the bull. When several velocity variables were measured, they collectively correlated with fertility and high $r^{2}$ values were obtained. However, Farrell et al. (1998) did not investigate the correlation between individual velocity parameters and fertility.

Using the data of the average velocity values (VCL, VSL, VAP) together with the NRR30 and PR75 values we found a close correlation with high significance between any one of the individual velocities and the non-return rates. Our results, in accordance with the observations of others, indicate that individual velocities can also be used to predict the fertilising potential of frozen-thawed semen (Byrd et al., 1990; Chan et al., 1990; Fetterolf and Rogers, 1990; Krause, 1995).

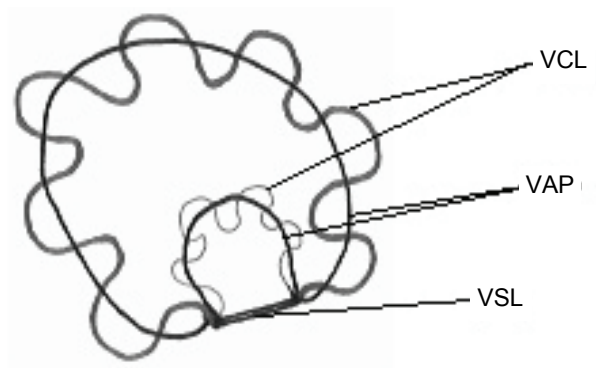

Fig. 4. Example of two tracks with different curvilinear velocity and average path velocity but the same straight-line velocity. VCL: curvilinear velocity, VSL: straight-line velocity, VAP: average path velocity

Following verification of the correlation between individual velocities and non-return rates, we compared one by one the results of VCL, VSL, VAP and found that VAP showed the highest correlation $[\mathrm{r}=0.7029(\mathrm{P}=0.023)$ for NRR30, $r=0.6652(P=0.036)$ for PR75]. This finding suggests that VAP may be the most useful semen motility characteristic (velocity parameter) which is of 
clinical relevance for predicting fertility, partly in contrast with previous research in the field that either was inconclusive (Amann, 1989) or was mainly concentrated on VCL as a parameter to predict fertility (Burkman, 1984; Hammond et al., 1986; Brown et al., 1988; Donnelly et al., 1998). As spermatozoa tracks with different characteristics (VCL, VAP) seem to have the same VSL velocity (Fig. 4), VAP should be preferred to evaluate and predict the fertilising potential of fresh or frozen-thawed bull semen. The equations presented above could be used in a clinical setting to predict the fertility potential of a semen sample by substituting $\mathrm{X}$ with the average VAP value of the semen in question.

In conclusion, increasing attention has been paid to the evaluation of different velocity parameters of spermatozoa besides simply determining the total proportion of motile spermatozoa and researchers started to investigate the relationship between velocity parameters and the fertilising ability of sperm. The introduction of CASA systems based on video images made it possible to measure the motility characteristics of individual spermatozoa. CASA allows to evaluate the fertilising capability of semen based on the velocity characteristics of spermatozoa. Based on our results, it can be concluded that VAP values generated by CASA could be used to predict the fertilising potential of frozen-thawed bull semen.

\section{Acknowledgements}

This study was supported by a project grant from the Hungarian Scientific Research Fund (OTKA project no. 17581/2010) and the Researcher Faculty Grant of the Faculty of Veterinary Science, Szent István University (KK-KF-2013).

\section{References}

Amann, R. P. (1989): Can the fertility potential of a seminal sample be predicted accurately? J. Androl. 10, 89-98.

Amann, R. P. and Hammerstedt, R. H. (1993): In vitro evaluation of sperm quality: an opinion. J. Androl. 14, 397-406.

Amann, R. P. and Waberski, D. (2014): Computer-assisted sperm analysis (CASA): capabilities and potential developments. Theriogenology 81, 5-17.

Andersson, M., Hellman, T., Holmstrom, B. G. and Jokinen, L. (1992): Computerized and subjective assessments of post-thaw motility of semen from Finnish Ayrshire AI bulls in relation to non-return rates. Acta Vet. Scand. 33, 89-93.

Auger, J., Leonce, S., Jouannet, P. and Ronot, X. (1993): Flow cytometric sorting of living, highly motile human spermatozoa based on evaluation of their mitochondrial activity. J. Histochem. Cytochem. 41, 1247-1251.

Barros, C., Fujimoto, M. and Yanagimachi, R. (1973): Failure of zona penetration of hamster spermatozoa after prolonged preincubation in a blood serum fraction. J. Reprod. Fertil. 35, 89-95. 
Bollendorf, A., Check, J. H. and Lurie, D. (1996): Evaluation of the effect of the absence of sperm with rapid and linear progressive motility on subsequent pregnancy rates following intrauterine insemination or in vitro fertilization. J. Androl. 17, 550-557.

Bratton, R. W., Foote, R. H., Henderson, C. R., Musgrave, S. D., Dunbar, R. S. Jr., Dunn, H. O. and Beardsley, J. P. (1956): The relative usefulness of combinations of laboratory tests for predicting the fertility of bovine semen. J. Dairy Sci. 39, 1542-1549.

Broekhuijse, M. L., Sostaric, E., Feitsma, H. and Gadella, B. M. (2011): Additional value of computer assisted semen analysis (CASA) compared to conventional motility assessments in pig artificial insemination. Theriogenology 76, 1473-1486 e1.

Broekhuijse, M. L., Sostaric, E., Feitsma, H. and Gadella, B. M. (2012): Application of computerassisted semen analysis to explain variations in pig fertility. J. Anim. Sci. 90, 779-789.

Brown, C. A., Boone, W. R. and Shapiro, S. S. (1988): Improved cryopreserved semen fecundability in an alternating fresh-frozen artificial insemination program. Fertil. Steril. 50, 825-827.

Budworth, P. R., Amann, R. P. and Chapman, P. L. (1988): Relationships between computerized measurements of motion of frozen-thawed bull spermatozoa and fertility. J. Androl. 9, 41-54.

Burkman, L. J. (1984): Characterization of hyperactivated motility by human spermatozoa during capacitation: comparison of fertile and oligozoospermic sperm populations. Arch. Androl. 13, 153-165.

Byrd, W., Bradshaw, K., Carr, B., Edman, C., Odom, J. and Ackerman, G. (1990): A prospective randomized study of pregnancy rates following intrauterine and intracervical insemination using frozen donor sperm. Fertil. Steril. 53, 521-527.

Centola, G. M. (1996): Comparison of manual microscopic and computer-assisted methods for analysis of sperm count and motility. Arch. Androl. 36, 1-7.

Chan, S. Y., Tsoi, W. L., Leung, J., Ng, V., Lo, T. and Wang, C. (1990): The accuracy of sperm concentration determination by the automated CellSoft semen analyzer before and after discontinuous Percoll gradient centrifugation. Andrologia 22, 55-61.

Chan, S. Y., Wang, C., Song, B. L., Lo, T., Leung, A., Tsoi, W. L. and Leung, J. (1989): Computer-assisted image analysis of sperm concentration in human semen before and after swim-up separation: comparison with assessment by haemocytometer. Int. J. Androl. 12, 339-345.

Christensen, P., Brockhoff, P. B. and Lehn-Jensen, H. (1999): The relationship between semen quality and the nonreturn rate of bulls. Reprod. Dom. Anim. 34, 503-507.

Coetzee, K., Kruger, T. F. and Lombard, C. J. (1999): Repeatability and variance analysis on multiple computer-assisted (IVOS) sperm morphology readings. Andrologia 31, 163-168.

Davis, A. P., Graham, J. K. and Foote, R. H. (1987): Homospermic versus heterospermic insemination of zona-free hamster eggs to assess fertility of fluorochrome-labeled acrosome-reacted bull spermatozoa. Gamete Res. 17, 343-354.

den Daas, N. (1992): Laboratory assessment of semen characteristics. Anim. Reprod. Sci. 28, 87-94.

Donnelly, E. T., Lewis, S. E. M., McNally, J. A. and Thompson, W. (1998): In vitro fertilization and pregnancy rates: the influence of sperm motility and morphology on IVF outcome. Fertil. Steril. 70, 305-314.

Dott, H. M. and Foster, G. C. (1979): The estimation of sperm motility in semen, on a membrane slide, by measuring the area change frequency with an image analysing computer. J. Reprod. Fertil. 55, 161-166.

Farrell, P. B., Presicce, G. A., Brockett, C. C. and Foote, R. H. (1998): Quantification of bull sperm characteristics measured by computer-assisted sperm analysis (CASA) and the relationship to fertility. Theriogenology 49, 871-879.

Fetterolf, P. M. and Rogers, B. J. (1990): Prediction of human sperm penetrating ability using computerized motion parameters. Mol. Reprod. Dev. 27, 326-331.

Freour, T., Jean, M., Mirallie, S. and Barriere, P. (2012): Computer-assisted sperm analysis parameters in young fertile sperm donors and relationship with age. Syst. Biol. Reprod. Med. 58, 102-106. 
Gerris, J. and Khan, I. (1987): Correlation between in vitro fertilization and human sperm density and motility. J. Androl. 8, 48-54.

Gunzel-Apel, A. R., Gunther, C., Terhaer, P. and Bader, H. (1993): Computer-assisted analysis of motility, velocity and linearity of dog spermatozoa. J. Reprod. Fertil. Suppl. 47, 271-278.

Hammond, M. G., Jordan, S. and Sloan, C. S. (1986): Factors affecting pregnancy rates in a donor insemination program using frozen semen. Am. J. Obstet. Gynecol. 155, 480-485.

Hirano, Y., Shibahara, H., Obara, H., Suzuki, T., Takamizawa, S., Yamaguchi, C., Tsunoda, H. and Sato, I. (2001): Relationships between sperm motility characteristics assessed by the computer-aided sperm analysis (CASA) and fertilization rates in vitro. J. Assist. Reprod. Genet. 18, 213-218.

Irvine, D. S., Macleod, I. C., Templeton, A. A., Masterton, A. and Taylor, A. (1994): A prospective clinical study of the relationship between the computer-assisted assessment of human semen quality and the achievement of pregnancy in vivo. Hum. Reprod. 9, 2324-2334.

Januskauskas, A., Gil, J., Soderquist, L., Haard, M. G., Haard, M. C., Johannisson, A. and Rodriguez-Martinez, H. (1999): Effect of cooling rates on post-thaw sperm motility, membrane integrity, capacitation status and fertility of dairy bull semen used for artificial insemination in Sweden. Theriogenology 52, 641-658.

Kathiravan, P., Kalatharan, J., Edwin, M. J. and Veerapandian, C. (2008): Computer automated motion analysis of crossbred bull spermatozoa and its relationship with in vitro fertility in zona-free hamster oocytes. Anim. Reprod. Sci. 104, 9-17.

Koops, W. J., Grossman, M. and Den Daas, J. H. (1995): A model for reproductive efficiency of dairy bulls. J. Dairy Sci. 78, 921-928.

Krause, W. (1995): Computer-assisted semen analysis systems: comparison with routine evaluation and prognostic value in male fertility and assisted reproduction. Hum. Reprod. 10, Suppl. 1, 60-66.

Larsen, L., Scheike, T., Jensen, T. K., Bonde, J. P., Ernst, E., Hjollund, N. H., Zhou, Y., Skakkebaek, N. E. and Giwercman, A. (2000): Computer-assisted semen analysis parameters as predictors for fertility of men from the general population. The Danish First Pregnancy Planner Study Team. Hum. Reprod. 15, 1562-1567.

Marshburn, P. B., McIntire, D., Carr, B. R. and Byrd, W. (1992): Spermatozoal characteristics from fresh and frozen donor semen and their correlation with fertility outcome after intrauterine insemination. Fertil. Steril. 58, 179-186.

Oliveira, L. Z., de Arruda, R. P., Cesar de Andrade, A. F., Carvalho Celeghini, E. C., Reeb, P. D., Nascimento Martins, J. P., dos Santos, R. M., Beletti, M. E., Guimaraes Peres, R. F., Monteiro, F. M. and Martins Hossepian de Lima, V. F. (2013): Assessment of in vitro sperm characteristics and their importance in the prediction of conception rate in a bovine timedAI program. Anim. Reprod. Sci. 137, 145-155.

Verstegen, J., Iguer-Ouada, M. and Onclin, K. (2002): Computer assisted semen analyzers in andrology research and veterinary practice. Theriogenology 57, 149-179.

World Health Organization (2010): WHO Laboratory Manual for the Examination and Processing of Human Semen. Fifth edition. WHO Press, Geneva, Switzerland.

Zhang, B. R., Larsson, B., Lundeheim, N. and Rodriguez-Martinez, H. (1998): Sperm characteristics and zona pellucida binding in relation to field fertility of frozen-thawed semen from dairy AI bulls. Int. J. Androl. 21, 207-216. 